\title{
Electron and photon energy measurement calibration with the ATLAS detector
}

\author{
Stefanie Morgenstern*, on behalf of the ATLAS Collaboration \\ Institut für Kern- und Teilchenphysik, Technische Universität Dresden, Dresden, Germany \\ CERN, Geneva, Switzerland \\ E-mail: stefanie.morgensternecern.ch
}

\begin{abstract}
An accurate calibration of the energy measurement of electrons and photons is needed for many ATLAS physics analyses. The calibration of the energy measurement is performed in-situ using a large statistics of $Z \rightarrow e^{-} e^{+}$events. A pre-requisite of this calibration is a good understanding of the material in front of the calorimeter and of the inter-calibration of the different calorimeter layers. The $Z \rightarrow e^{-} e^{+}$sample is also used to measure the energy resolution. The results obtained with proton-proton collision data at $\sqrt{s}=13 \mathrm{TeV}$ in 2015-2017 corresponding to an integrated luminosity of $80 \mathrm{fb}^{-1}$ are presented as well as the corresponding uncertainties on the electron and photon energy scales.
\end{abstract}

The 39th International Conference on High Energy Physics (ICHEP2018)

4-11 July, 2018

Seoul, Korea

${ }^{*}$ Speaker. 


\section{Introduction}

The ATLAS detector [1] is a multi-purpose detector at the Large Hadron Collider (LHC) at CERN. It is designed to measure the particles and their decay products created in proton-proton collisions. During the ongoing Run-2 (2015 - 2018) a center-of-mass energy of $\sqrt{s}=13 \mathrm{TeV}$ and a peak luminosity of $2.14 \times 10^{34} \mathrm{~cm}^{-2} \mathrm{~s}^{-1}$ have been reached. During the past three years more than $100 \mathrm{fb}^{-1}$ of data have been collected by the ATLAS experiment and are available for physics analyses. Many of these analyses target final states involving electrons and photons and thus rely on an excellent reconstruction, identification and calibration of these particles. Especially an accurate energy calibration is crucial for various precision measurements.

The energy of electrons and photons is measured by the liquid-argon calorimeter. The traversing particles induce an electromagnetic shower and thus the ionisation of the liquid argon. The ionisation electrons drift to the read-out electrodes due to the high voltage applied in the liquid argon gap. The induced signal is amplified, shaped, digitised and translated into the energy measured in single calorimeter cells. These cells are combined to build clusters which can be identified as electrons and photons. The cluster energy is then calibrated following the procedure summarised in Fig.1a.

\section{Simulation based calibration}

The first aspect of the energy calibration aims to recover the energy loss outside of the cluster and in passive material. Therefore, a multivariate approach in form of a regression tree with gradient boosting (BDT) is chosen. A set of variables sensitive to the energy response is used to train on simulated single electrons and photons targeting the ratio of the simulated and reconstructed energy. The multivariate calibration is then applied to data as well as simulation. The input variable set is further optimised for converted photons and in the transition region between the barrel and the endcap calorimeters at $|\eta| \sim 1$.5. A more detailed description can be found elsewhere [3].

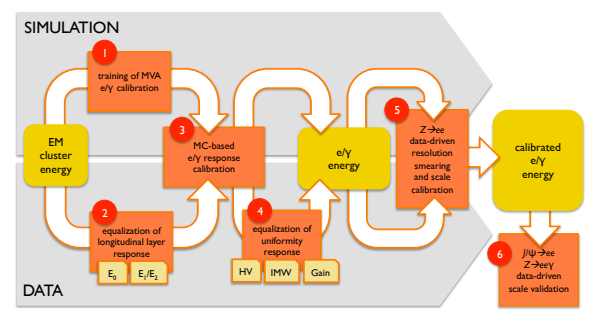

(a)

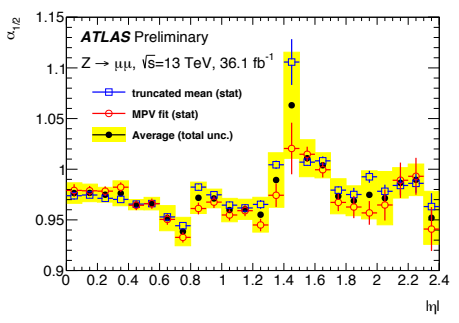

(b)

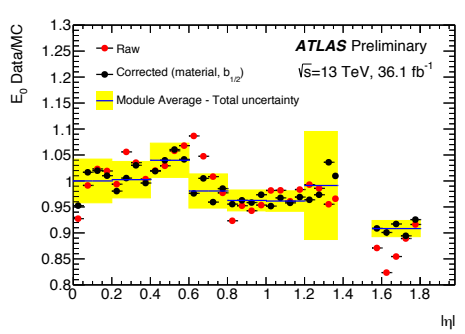

(c)

Figure 1: Schematic overview of the calibration chain (a) [2]. $\alpha_{1 / 2}$ (b) and $\alpha_{P S}$ (c) as a function of $|\eta|$ (see text for definitions) [5].

\section{Data-driven corrections}

Besides the simulation based calibration, data-driven corrections are put in place to mitigate the non-uniformity in the detector response. 


\section{Inter-layer calibration and presampler calibration}

Mis-calibration of the calorimeter layer response impacts the overall energy measurement. To account for this an inter-layer calibration of the first and second layer is undertaken by comparing the energy deposits of muons in data and simulation. A correction factor $\alpha_{1 / 2}$ is extracted as a double ratio of the energy deposits in the first and second calorimeter layer in data and simulation. This correction is derived in several bins in $\eta$ (Fig.1b) and applied on the energy measurement of the second layer. The uncertainties on $\alpha_{1 / 2}$ are below $3 \%$.

A similar approach is chosen for the presampler which covers the central part of the electromagnetic calorimeter up to $|\eta|=1.8$. In order to determine the presampler energy scale, $\alpha_{P S}$, electrons are exploited to calculate the ratio of the energy deposited in the presampler $\left(E_{0}\right)$ in data and simulation whereby correction factors accounting for material variations in front of the presampler are considered as well. This correction is derived in several bins in $\eta$ and applied on the energy measurement of the presampler (Fig.1c). The uncertainties on $\alpha_{P S}$ are below $5 \%$.

\section{Uniformity corrections}

The liquid argon gaps in-between the 16 barrel modules are slightly larger than the gaps within the modules causing an underestimation of the measured energy in these regions. The gaps are further altered due to gravitation. This has been studied in $Z \rightarrow e^{-} e^{+}$events and a correction has been derived to account for the degradation in the energy measurement. Besides this a deviation in the measured energy can originate from high voltage zones which are not operated at the nominal value. At first order this is already corrected on reconstruction level but a remaining degradation can be observed in the invariant mass of $Z \rightarrow e^{-} e^{+}$events. Based on this, a correction factor is extracted for the affected $\eta-\phi$ regions. Both correction factors are applied to the energy measurement in data and recover the uniformity in $\eta$ and $\phi$. The impact on the resolution is of the order of $1 \%$ for each.

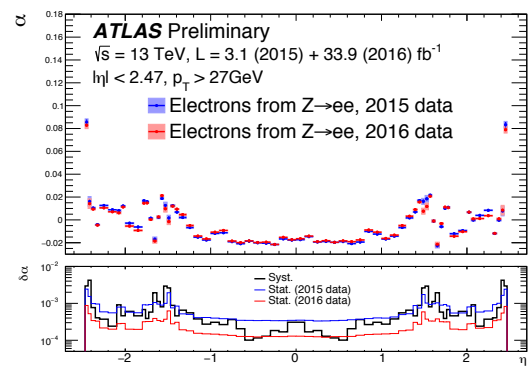

(a)

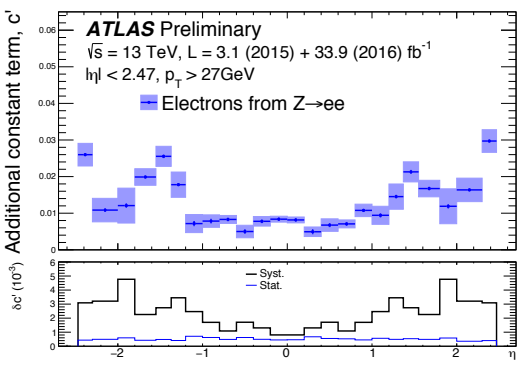

(b)

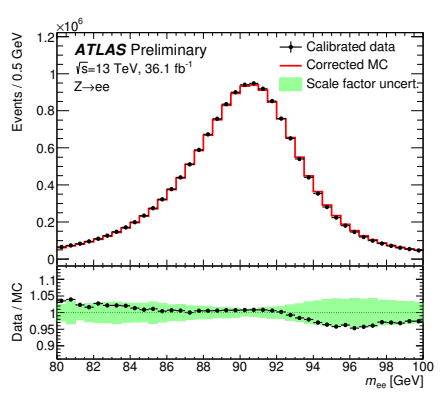

(c)

Figure 2: Scale (a) and resolution (b) of the calorimeter energy measurements [4]. Inclusive dielectron invariant mass distribution (c) from $Z \rightarrow e^{-} e^{+}$decays in data compared to simulation after applying the full calibration [5].

\section{Energy scale and resolution}

After the application of the simulation based calibration and data-driven corrections, a residual disagreement in the energy scale and resolution may be present between simulation and data. The 
energy mis-calibration between the measured, $E^{\text {data }}$, and simulated, $E^{\mathrm{MC}}$, energies is defined as $E_{i}^{\text {data }}=E_{i}^{\mathrm{MC}}\left(1+\alpha_{i}\right)$, where $\alpha_{i}$ represents the deviation from the optimal calibration and the index $i$ indicates a given pseudo-rapidity region. The relative energy resolution is parameterised as $\frac{\sigma(E)}{E}=$ $\frac{a}{\sqrt{E}} \oplus \frac{b}{E} \oplus c$, where $a$ is the sampling term related to shower fluctuations, $b$ the electronic noise term and $c$ the constant term. Parameters $a$ and $b$ can be extracted from simulation and calibration runs, respectively. The constant term needs to be extracted directly from data under the assumption that simulation models data well up to an effective constant term $c^{\prime}$. Both, the scale $\alpha$ and the effective constant term $c^{\prime}$ can be derived with a template fit method from $Z \rightarrow e^{-} e^{+}$events (Fig.2a, 2a). The scale $\alpha$ is applied on data and the resolution $c^{\prime}$ on simulation (Fig.2c).

Based on the energy dependence of the calibration and the disagreement of data and simulation, a set of systematic uncertainties is derived separately for electrons, converted and unconverted photons. Scale uncertainties reach a few per-mill. The main contributions arise from the layer-inter calibration, non-linearity of the cell energy measurement and material in front of the calorimeter. The main contributors to the resolution uncertainties are residual non-uniformities, fluctuation of the energy loss in front and in the calorimeter and impact of pile-up and electronics noise.

\section{Validation}

The calibration and corrections rely on electrons originating from $\mathrm{Z}$ decays and the scale and resolution are extrapolated to high energies and photons. In order to validate the reliability of this extrapolation, residual scales are extracted from $J / \Psi \rightarrow e^{-} e^{+}$and $Z \rightarrow l^{-} l^{+} \gamma(l=e, \mu)$ events after the full calibration chain and all corrections are applied. Based on $J / \Psi \rightarrow e^{-} e^{+}$an agreement within $1 \%$ was confirmed for low energy electrons. For photons from radiative $Z$ events an overall agreement within $0.3 \%$ is achieved.

\section{Summary}

The energy calibration of electrons and photons measured with the ATLAS detector shows an excellent performance so far during Run-2 of the LHC. The precise knowledge of the energy scale and resolution is a crucial ingredient for many physics analyses, both searches for new physics and precision measurements. The so far analysed $13 \mathrm{TeV}$ data reveals an excellent performance over a wide energy range. This is thanks to a continuous effort to improve the energy calibration of electrons and photons.

\section{References}

[1] ATLAS Collaboration, The ATLAS Experiment at the CERN Large Hadron Collider, JINST 3 S08003.

[2] ATLAS Collaboration, Electron and photon energy calibration with the ATLAS detector using LHC Run 1 data, Eur. Phys. J. C74 3071 [hep-ex/1407.5063].

[3] ATLAS Collaboration, Electron and photon energy calibration with the ATLAS detector using data collected in 2015 at $\sqrt{s}=13 \mathrm{TeV}$, ATL-PHYS-PUB-2016-015.

[4] ATLAS Collaboration, https://atlas.web.cern.ch/Atlas/GROUPS/PHYSICS/PLOTS/EGAM-2017-002.

[5] ATLAS Collaboration, https://atlas.web.cern.ch/Atlas/GROUPS/PHYSICS/PLOTS/EGAM-2018-001. 